\author{
دراسة اقتصادية لأثر التوسع فى البيوت المحمية على إنتاج القلفل الأخضر

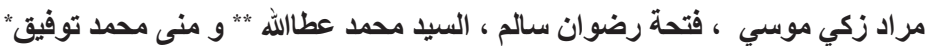

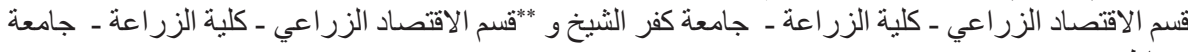

تعد البيوت الدحمية أهم وسائل زيادة الإنتاج، حيث تحقق زيادة ربح المز ارع مقارنة بالحقول المكثوفة، إضافة إلى أنها أحد

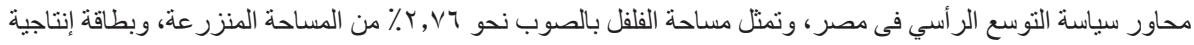

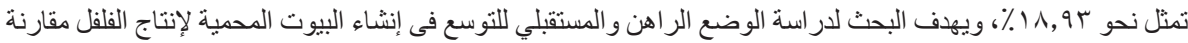

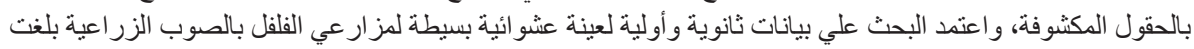

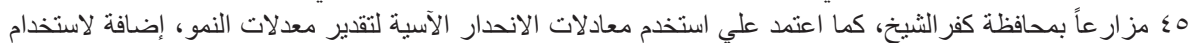

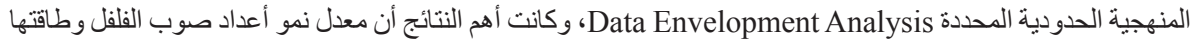

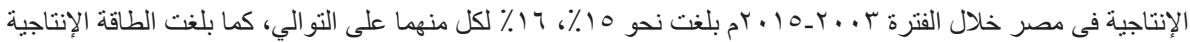

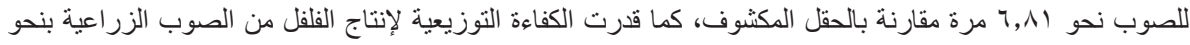

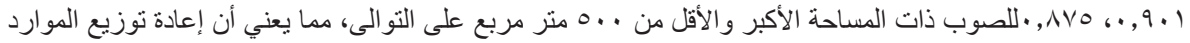

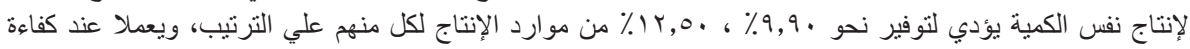

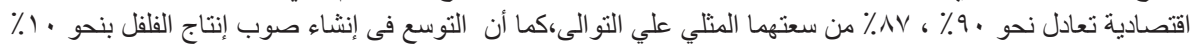

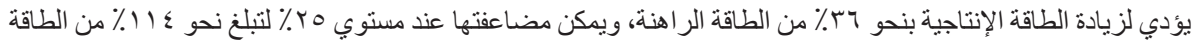

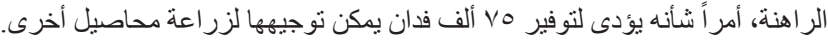

الكلمات الإفتتاحية: البيوت المحمية، تحليل مغلف البيانات، الكفاءة الاقتصادية، إنتاج الفلفل من البيوت المحمية

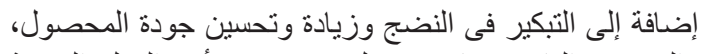

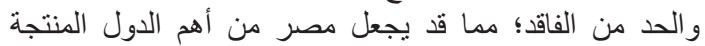

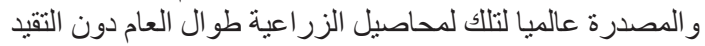

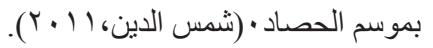

وتحقيقاً لهذا الهدف اهتم هذا البحث بالصوب الزراعية

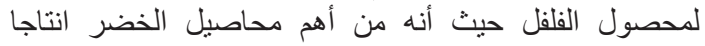

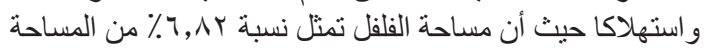

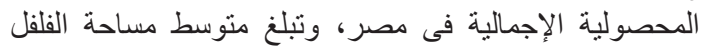

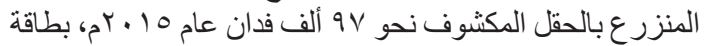

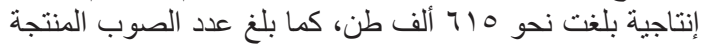

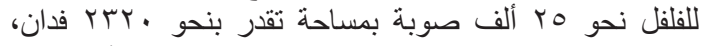

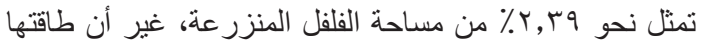

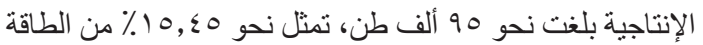

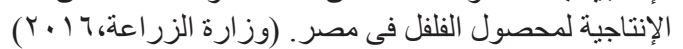

المشكيلة البمثة

في ظل التحديات التي تواجه التنمية بالقطاع الزراعي

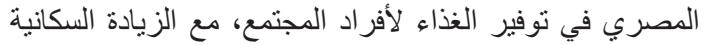

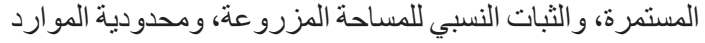

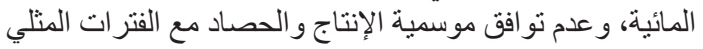

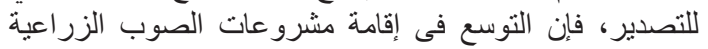

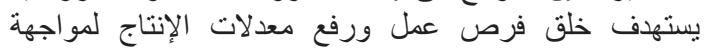

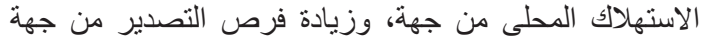

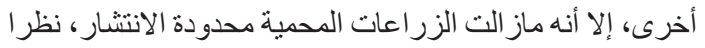

المقدمة

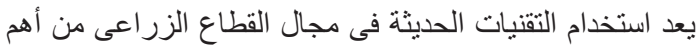
وسائل زيادة الإنتاج ورفع معدلات التئة التنمية الزراعية الزراعي، وتمثل الفثل

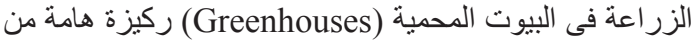

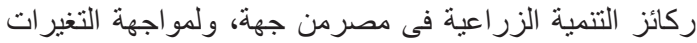

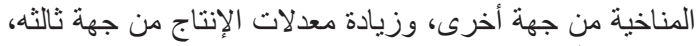

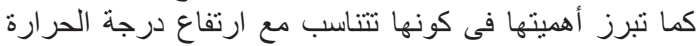

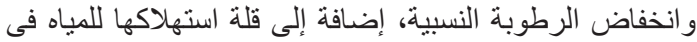

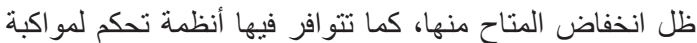

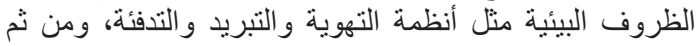

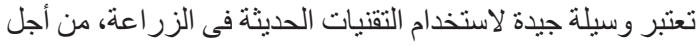

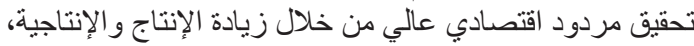

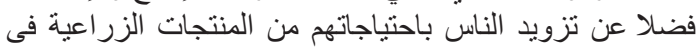

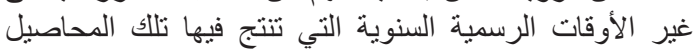

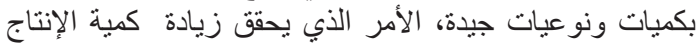

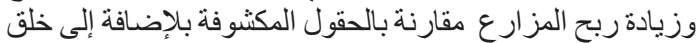

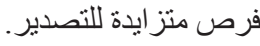

وتسعى الدولة للتوسع فى إنشاء هذه البيوت المحمية كأحد

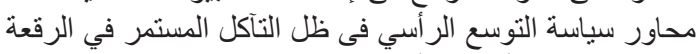

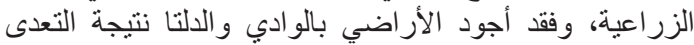

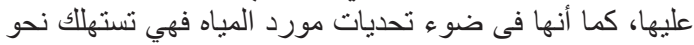

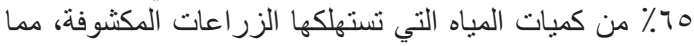

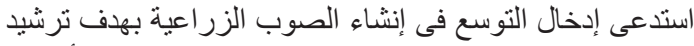
استهلاك المياه، و الحد من نمو الحشائش، وتوفير الزير كميات الأسمدة،

"Corresponding author: maiarali2010@yahoo.com

DOI : 10.21608/jsas.2018.5038.1092

C2018 National Information and Documentation Center (NIDOC) 


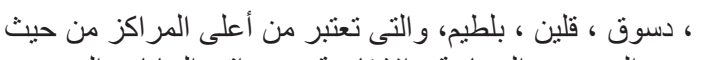

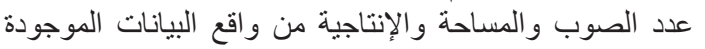

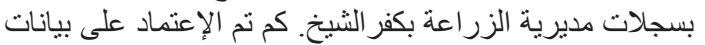

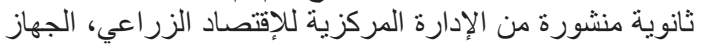
المركزي للتعبئة العامة و الإحصاء.

أولاً: الأهية النبية النسبية لنطور الطاقة الإنتاجية لصوب الفلفل

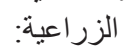

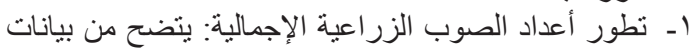

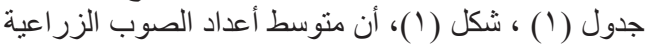

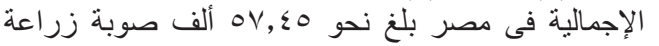

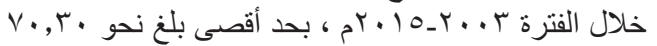

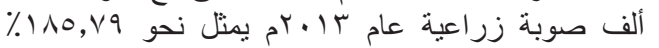

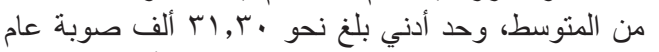

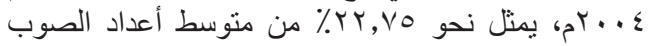

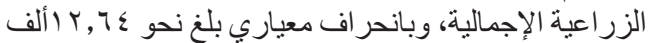

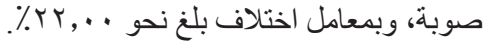

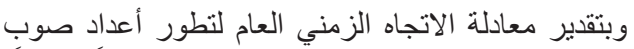

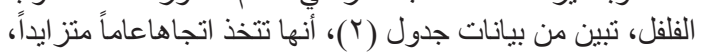

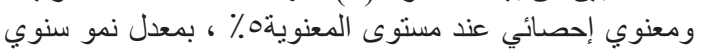

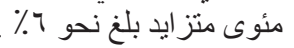

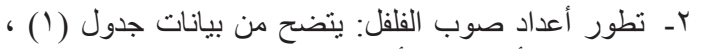

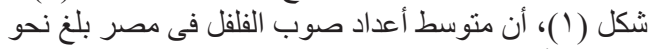

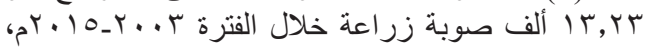

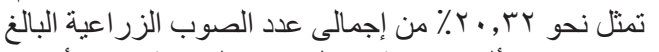

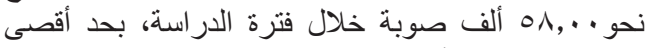

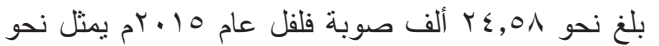

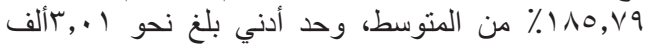

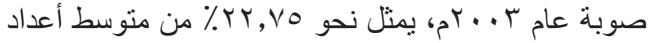

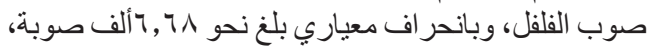

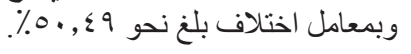

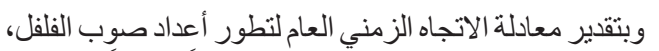

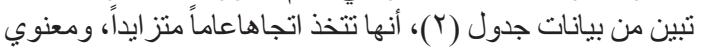

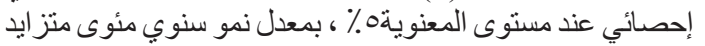
بلغ نحو 10 1 ، وهو أعلي من معدل النمو السنوي المئوي لأعداد

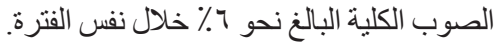

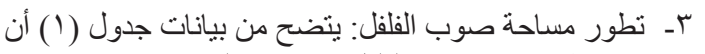

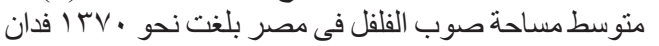

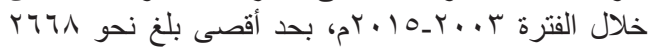

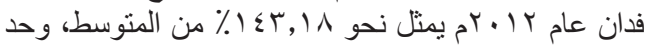

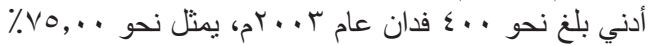

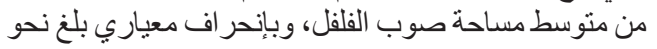

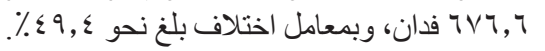

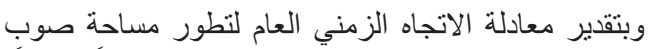

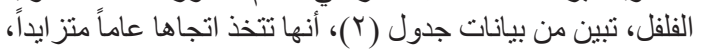

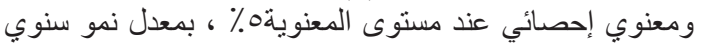

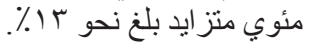

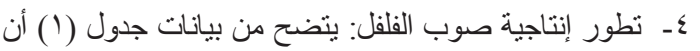

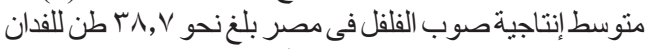

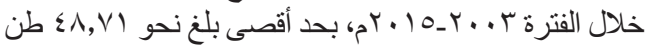

لارتفاع تكاليفها الاستثمارية على الرغم من تحقيقها عائداً وربحاً

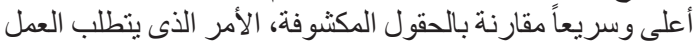

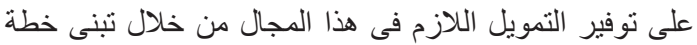

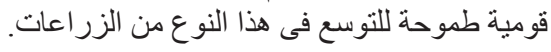

وقد نم اختيار محصول الفلفل بنظام الصوب الزر اعية بمحافظة

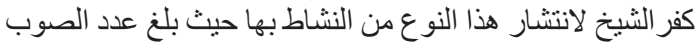

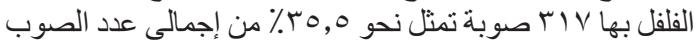

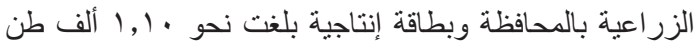

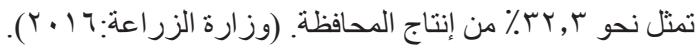

الأهداف البحثية

يستهدف هذا البحث مايلى: التئ

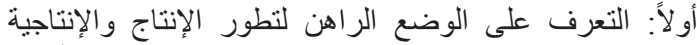

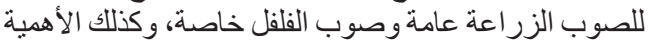

النسبية للطاقة الإنتاجية للصوبة مقاربة لـنة بالحقل المكثوف.

ثانياً: دراسة العائد للسعة لصوب الفلفل من خلال تقدير الأثر الإقتصادى لزيادة مساحة الصوبة.

ثالثاً: تقدير الكفاءة الفنية والتوزيعية والاقتصادية لإنتاج الفلفل من

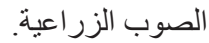

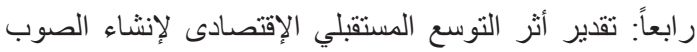
الزر اعية لإنتاج الفلفل على حساب الحقول المكثوفة.

الأسلوب البحثي

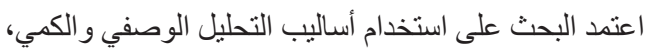

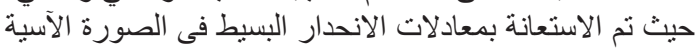

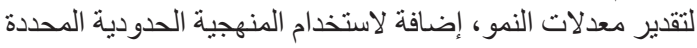
The Deterministic Frontier Approach

Data Envelopment Analysis تحليل مغلف البيانات لتقدير الكفاءة الاقتصادية، وهو أحد أساليب التحليل

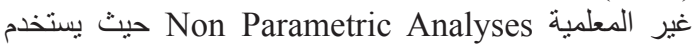
أسلوب البرمجة الخطية لإنشاء مجال يحوي التوليفات الفعلية

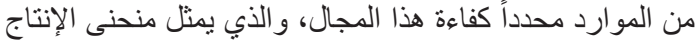

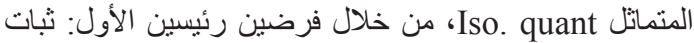
العائد للسعة (CRS) تعمل المنشأة عند طاقتها الإنتاجية القصوى، بينما الثاني: تغير

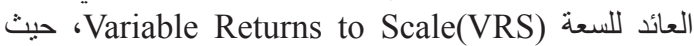
تعمل المنشأة عند مستوي أقل من طاقتها القصوى، مما يسمح

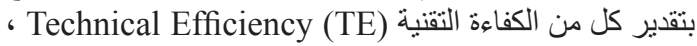
وكفاءة التكاليف Cost Efficiency (CE)، ولكفة والكفاءة التوزيعية Allocative Efficiency (AE)

(Moussa، १९९). Economic Efficiency (EE)

مصادر البيانات

اعتمدت الدر اسة علي بيانات أولية لمحصول الفلفل الأخضر

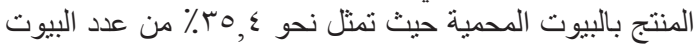

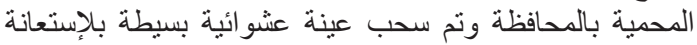

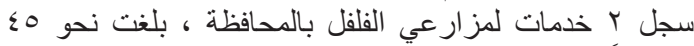

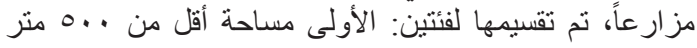

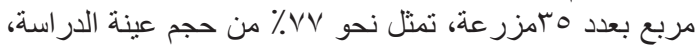

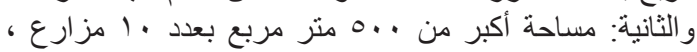
تمثل نحو بr\% من حجم عينة الدر اسة، وذلك بمر اكز كفر الثيخ 


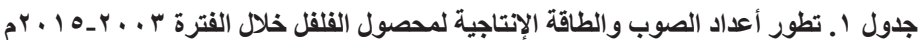

\begin{tabular}{|c|c|c|c|c|c|c|c|}
\hline \multirow{2}{*}{ ألف طن } & \multicolumn{2}{|c|}{ الإنتاجية } & \multirow{2}{*}{ مساحة صوب القلقل (فدان) } & \multicolumn{3}{|c|}{ عدد الصوب (ألف صوية) } & \multirow[b]{2}{*}{ السنوات } \\
\hline & متر مربع & (طن / فدان) & & $\%$ & 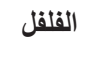 & إجمالي & \\
\hline 11,174 & $v, \ldots$ & $r q, \varepsilon$. & ५৭१,q. & $V, 9 Y$ & $r, \cdot$, & $r \wedge, .$. & $r \ldots r$ \\
\hline $1 \varepsilon, \wedge Y$ & $\gamma, O$. & r.,o. & $\varepsilon V \cdot, \varepsilon \leqslant$ & $1 r, 00$ & $\varepsilon, Y \varepsilon$ & r., r. & $r \ldots \varepsilon$ \\
\hline$r \Lambda, r V$ & $9, \ldots$ & TV,A. & $\mathrm{Vor}, \mathrm{O}$. & 17, 17 & $v, q$. & $\leqslant v, 0$. & r...o \\
\hline r,A. & $9, \leqslant 7$ & rq, vo & $\vee १ ৭, q \leq$ & 10,7 & $V, \infty 0$ & $\varepsilon \wedge, \varepsilon$. & $r \ldots r$ \\
\hline $0 \cdot, \wedge Y$ & $9, \vee 7$ & $\varepsilon \cdot, 91$ & $|r \varepsilon|, r r$ & $r \cdot, r)$ & 11,07 & $O V, r$. & $r \ldots v$ \\
\hline $79, Y 7$ & $\mid \cdot, v 1$ & $\varepsilon \varepsilon, 9 \vee$ & $10 \leq \cdot, 11$ & Yr,OT & $I \varepsilon, \wedge V$ & $70,9$. & $r \cdots \lambda$ \\
\hline $0 \leqslant, 0$. & $1 \cdot, 10$ & $\{r, T\}$ & $1 r \vee q, \cdot 1$ & $r, T \Lambda$ & $|r, T|$ & 00,7 & $r \ldots q$ \\
\hline or, 11 & $q, r v$ & rq, ro & 1 rol,or & $Y \cdot, T V$ & $1 r, 01$ & $70, v$. & $r \cdot 1$. \\
\hline$r_{4, \varepsilon r}$ & 0,9 . & $r \leqslant, V T$ & $1 \leq V \cdot, \wedge T$ & $19, \wedge \mathrm{V}$ & $I Y, \leqslant \wedge$ & $T r, \wedge$. & $r .11$ \\
\hline $9 V, 7 V$ & $\Lambda, \vee r$ & (1) & rTTV,90 & $r 7, \cdot 9$ & 18,9 . & $\uparrow \wedge, \uparrow$ & $r+1 r$ \\
\hline$V V, \ldots$ & $11,7$. & $\{\wedge, \vee \backslash$ & $101 \cdot, V Y$ & $r 0, V r$ & $11, \cdot 9$ & $v \cdot r . r$. & $r \cdot 1 r$ \\
\hline$\lambda V, \Sigma V$ & $1 \cdot, \mathrm{AV}$ & $\{0,70$ & $1917,1 \leq$ & $r \varepsilon, V)$ & Tr,TV & $T \Lambda, r$. & $r \cdot 1 \leq$ \\
\hline $1 \cdot V, 1 v$ & $1 \cdot, 9$. & $\varepsilon 0, v \wedge$ & $0 r r \leqslant \cdot, q$ & rq,OT & $r \leq, O N$ & $T V, r$. & $r .10$ \\
\hline $0 \leqslant, Y \leqslant$ & $9, r$. & $\Gamma \Lambda, T \Lambda$ & $1+49,90$ & $r \cdot, r r$ & IT,YT & $O V, \leqslant 0$ & المتوسط \\
\hline $1 \cdot V, I V$ & 11,7 & $\varepsilon \wedge, \vee \uparrow$ & rTYV,90 & r T,Or & $r \leq, 0 \wedge$ & $v \cdot r \cdot r \cdot$ & حدالأفصى \\
\hline 11,174 & 0,9 . & $r \leq, V T$ & rq৭,q. & $V, q Y$ & $r, \cdot 1$ & $\mu, r$. & حدالأننى \\
\hline$r \wedge, q \vee$ & 1,71 & $\uparrow, \vee \wedge$ & TVY, T. & $V, 9 Y$ & $7,7 \uparrow$ & $1 Y, 7 \varepsilon$ & ل انحرافـمعياري \\
\hline or, \&1 & $1 \Lambda, \cdot \varepsilon$ & IV,or & $\varepsilon q, r q$ & $r \wedge, 97$ & $0 ., \leqslant 9$ & $r, \ldots$ & معلملاختالف \\
\hline
\end{tabular}

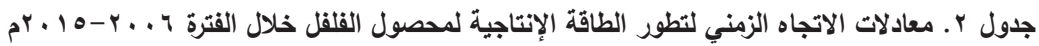

\begin{tabular}{|c|c|c|c|c|}
\hline $\mathbf{F}$ & $\mathbf{R}^{r}$ & معدل النمو السنوي المئوي ٪ & المعادلة & المتغيرات \\
\hline$r \cdot, 10 \%$ & שTr, & $7, \cdots$ & $\begin{array}{l}Y=e^{r, 7 \varepsilon+\ldots, \cdot X} \\
(\varepsilon 0, r Y)^{\bullet}(0, \varepsilon q)^{*}\end{array}$ & أعداد الصوب الزراعية (ألف صوبة) \\
\hline$T V, r V *$ & $\cdot, \wedge \uparrow$. & $10, \ldots$ & $\begin{array}{l}Y=e^{1, r 9+\cdot, 10 X} \\
\left.(9, r \cdot)^{*}(\lambda, r)\right)^{*}\end{array}$ & أعداد صوب الفلفل (ألف صوبة) \\
\hline $01,99 \%$ & $\cdot, \wedge$ YO & $1 r, \ldots$ & $\begin{array}{l}Y=e^{i, 10+., 1 r X} \\
(\xi 1, T Y)^{\circ} \quad(Y, Y Y)^{\circ}\end{array}$ & مساحة صوبة الفلفل مترمربع \\
\hline r,vq & $\cdot, r \cdot r$ & $r, \cdots$ & $\begin{array}{l}Y=e^{r, \xi \lambda+\ldots, r X} \\
\left.(r r, r Y)^{*}(1, T \gamma)\right)\end{array}$ & الإنتاجية (طن / فدان) \\
\hline$r \Lambda, V \Gamma^{*}$ & $\cdot, \vee \vee q$ & $17, \ldots$ & $\begin{array}{l}Y=e^{r, Y r+\cdot, 1 \eta X} \\
(1 r, Y \xi)^{\bullet} \quad(7, Y r)^{*}\end{array}$ & الإنتاج (ألف طن) \\
\hline
\end{tabular}

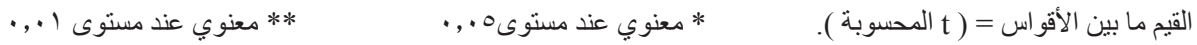

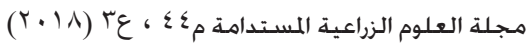

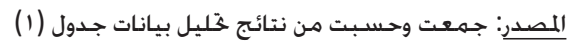




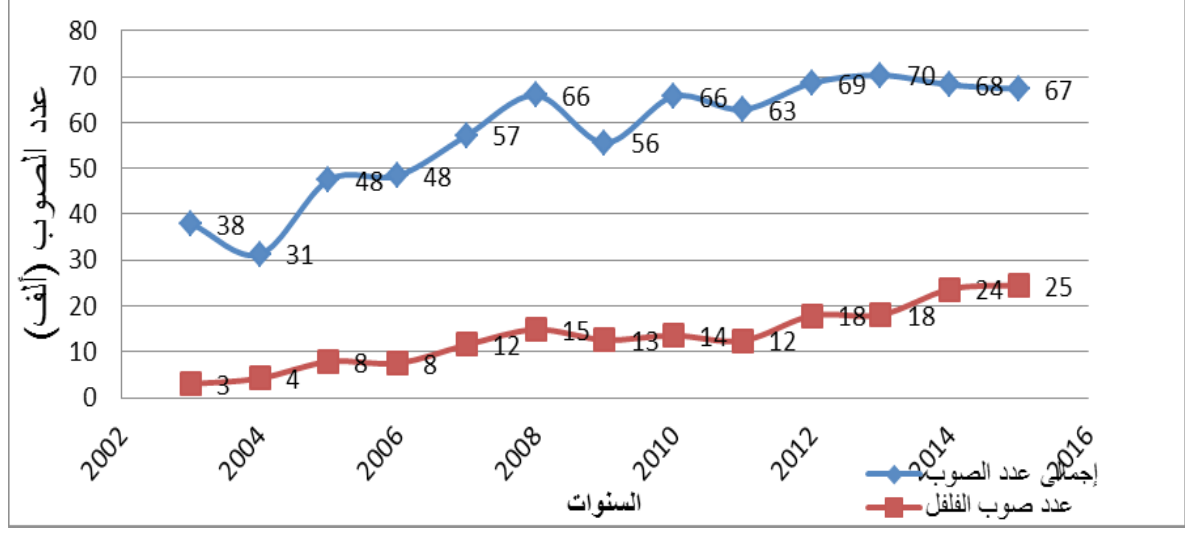

شكل 1 ـ تطور اجمالى اعداد الصوب الزراعية و عدد صوب القلقل

المصدر: بيانات وزارة الزر اعة واستصلاح الأراضي: الإدارة المركزية للاقتصاد الزر اعي ـ نشرة الاقتصاد الزر اعي ـ أعداد متفرقة

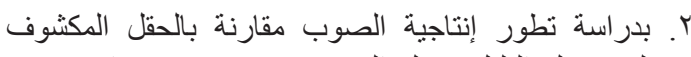

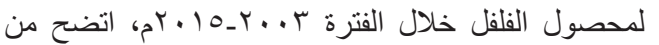

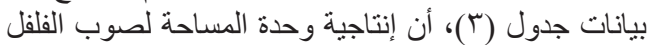

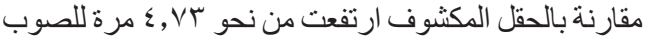

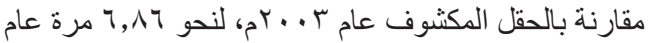

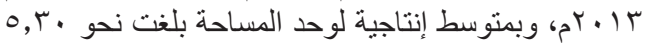

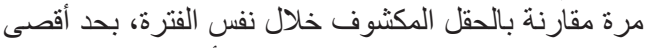

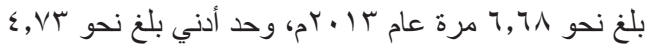

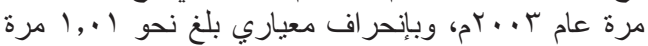
لصوب الفلفل مقارنة بالحقل المكثوف.

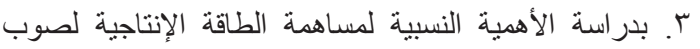

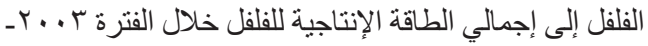
10

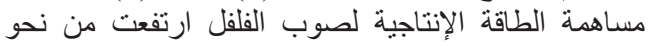

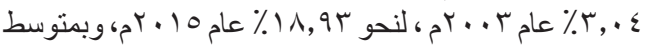

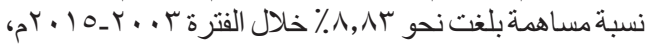

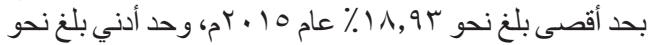

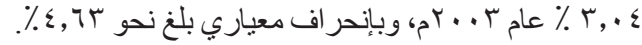

ثالثاً: تقدير الكفاءة الفنية والتوزيعية والاقتصادية لإنتاج الفلفل من الصوب الزر اعية باستخدام طريقة تحليل مغلف اللئل البيانات DEA

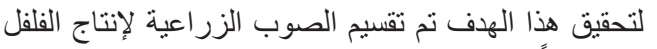

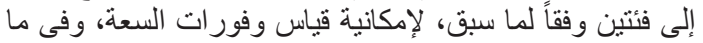

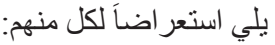

أ. تقدير الكفاءة الفنبة لإنتاج محصول الفلفل من الصوب باستخدام

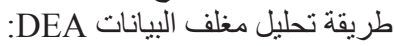

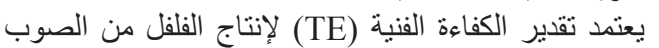

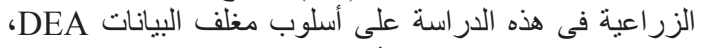

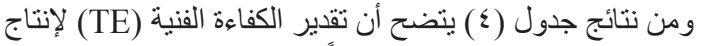

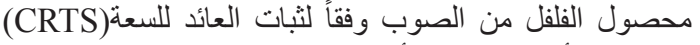

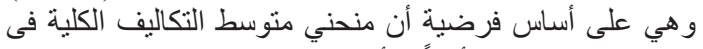

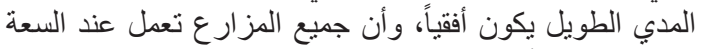

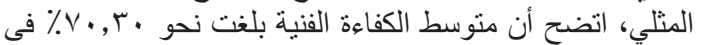

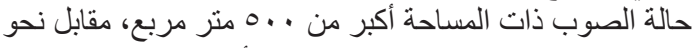

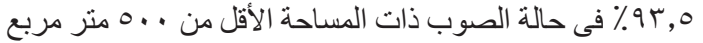
في ظل افتر اض ثبات العائد للسعة.

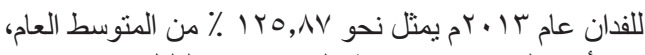

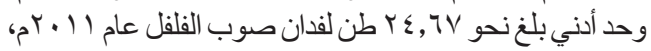

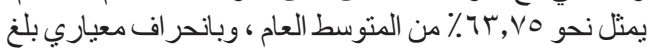

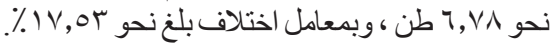

وبتقدير معادلة الاتجاه الزمني العام لتطور إنتاجية صوب إناب

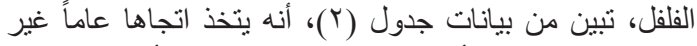

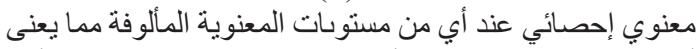

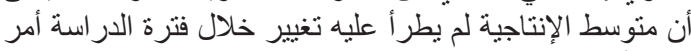

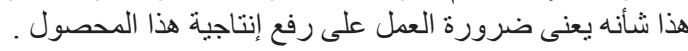

0ـ نطور الطاقة الإنتاجية لصوب الفلفل: يتضح من بيانات جدول فئل

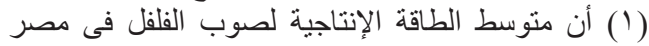

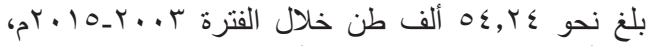

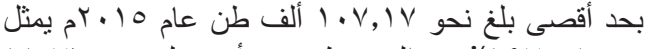

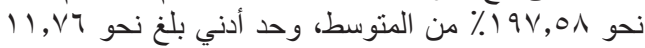

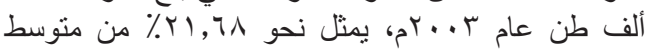

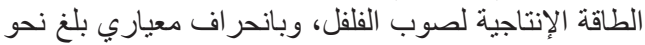

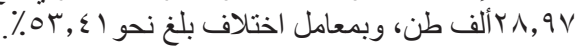

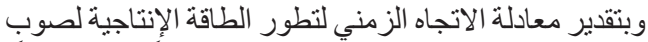

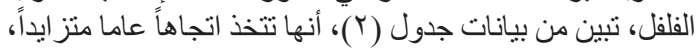

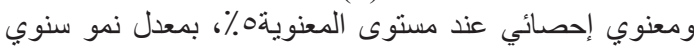

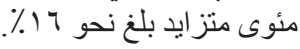

ثانياً: الأهمية النسبية للطاقة الإنتاجية للصوب مقارنة بالحقل المكثوف لمحصول الفلفل.

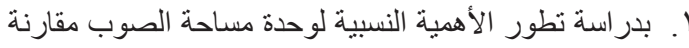

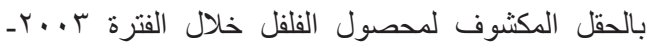

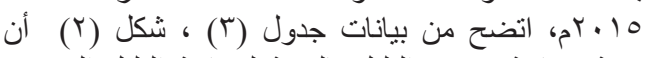

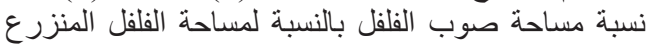

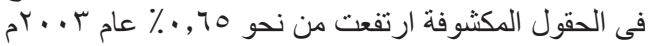

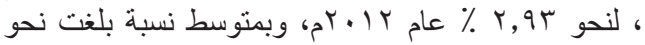

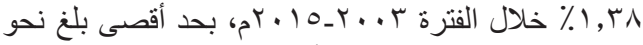

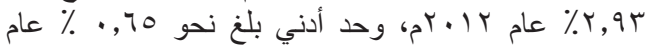

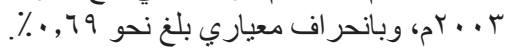

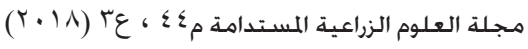




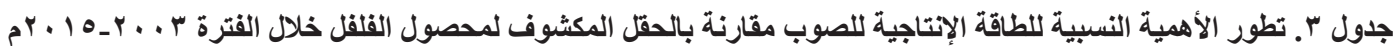

\begin{tabular}{|c|c|c|c|}
\hline الطاقة الإنتاجية للصوب . & بالنسبة للمكشوف (مرة) & 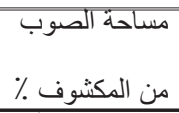 & السنوات \\
\hline$T, \cdot \varepsilon$ & $\varepsilon, V T$ & $\cdot, 70$ & $T \cdots T$ \\
\hline$T, 11$ & $\varepsilon, V T$ & $\cdot, 70$ & T.? \\
\hline $0, V 1$ & $0, \mathrm{VO}$ & $\cdot, 99$ & T.. \\
\hline $7, \lambda 1$ & $7, .1$ & $1,1 T$ & $T \cdots T$ \\
\hline $9, \cdot \varepsilon$ & $7,+7$ & 1,00 & T.V \\
\hline $9,7 \pi$ & 7,07 & $1,0 \varepsilon$ & $r \cdots 1$ \\
\hline$\lambda, r^{\prime \prime}$ & T,YY & T, ro & T..9 \\
\hline 7,19 & 0,70 & $T, \Gamma \varepsilon$ & $T \cdot 1 \cdot$ \\
\hline$\varepsilon, 09$ & r, rq & $1, r^{4}$ & $T \cdot 11$ \\
\hline$T, V \lambda$ & 0,11 & T,QT & $T \cdot I T$ \\
\hline$\Pi, \varepsilon 9$ & $7, \wedge \uparrow$ & T,OY & $T \cdot T T$ \\
\hline T, ह ह & 7,70 & 1,97 & $T \cdot 1 \varepsilon$ \\
\hline T1,9T & $7, \lambda 1$ & T,V7 & $T \cdot 10$ \\
\hline$\lambda, \lambda \Gamma$ & $0, V T$ & $T, \Gamma \wedge$ & المتوسط \\
\hline 11,94 & 7,17 & $T, 9 T$ & الحـد الأقصى \\
\hline$T, \cdot \varepsilon$ & T, rq & $\cdot, 70$ & الحـ الأدنى \\
\hline$\varepsilon, 7 T$ & $1, \cdot 1$ & $\cdot, 79$ & الإنحراف المعيارى \\
\hline OY, हV & $T V, 70$ & $0 ., Y \wedge$ & معامل الاختلاف \\
\hline
\end{tabular}

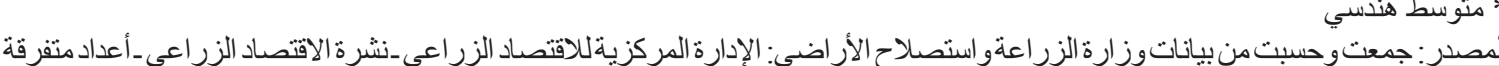

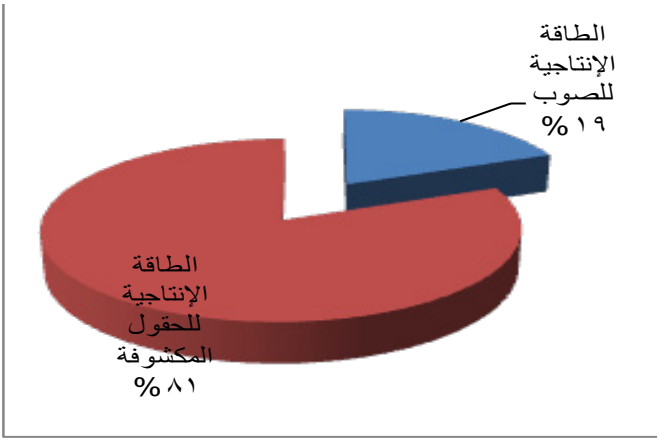

شكل r ـ الأهمية النسبية للطاقة الإتتاجية لصوب الفلفل للحقول المكشوفة

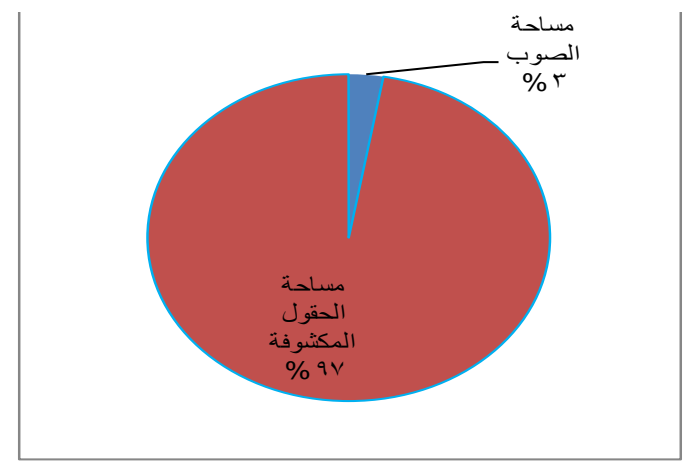

شكل ץ ـ الأهمية النسبية لمساحة صوب الفلقل للحقول المكثوفة جدول ؛ ـ نتائج تقدير الكفاءة الفنية والتوزيعية والاقتصادية لأثر اختلاف سعة الصوب المزرعية على إنتاجية محصول الفلفل

\begin{tabular}{|c|c|c|c|}
\hline فئات أقل من . .ـ متر ؟ & فئات أعلى من . .. متر r & \multicolumn{2}{|c|}{ المتفيرات } \\
\hline$\cdot, 9 \leftrightarrow q$ & $\cdot, V \cdot r$ & CRS & \multirow{2}{*}{ لكفاءة الفنية Te } \\
\hline$\cdot, 99$. & $1, \cdots$ & VRS & \\
\hline$\cdot, 9 \leq \wedge$ & $\cdot, v \cdot r$ & \multicolumn{2}{|c|}{ SEفاءة السعة SE } \\
\hline$\cdot, \wedge \uparrow \wedge$ & $\cdot, 9 \cdot 1$ & \multicolumn{2}{|c|}{ كفاءة التكاليف CE } \\
\hline$\cdot, \wedge \vee 0$ & $\cdot, 9 \cdot 1$ & \multicolumn{2}{|c|}{ AE الكفاءة التوزيعية } \\
\hline דזה,י & $\cdot, 9 \cdot 1$ & \multicolumn{2}{|c|}{ الكفاءة الاقتصادية EE } \\
\hline
\end{tabular}

WINDEAPr, المصدر: جمعت وحسبت من تحليل البيانات للعينة البحثية باستخدام برنامت 


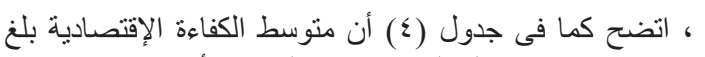

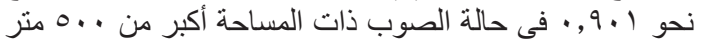

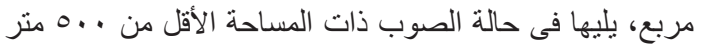

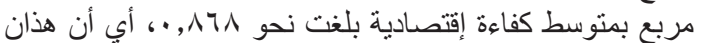

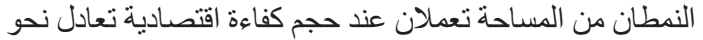

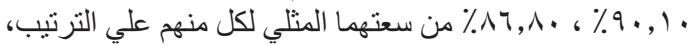

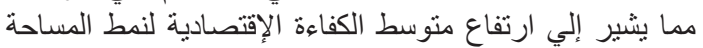
الأكبر فى صوب إنتاج الفلفل.

رابعا: تقدير متوسط كمية وقيمة الفائض للمدخلات الإنتاجية

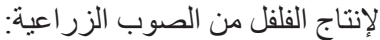

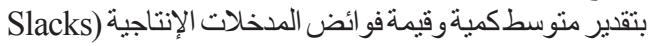
(Input

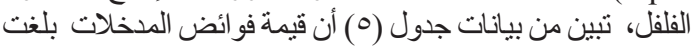

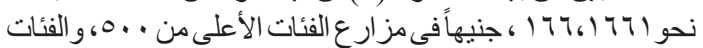

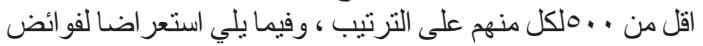

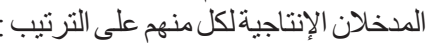

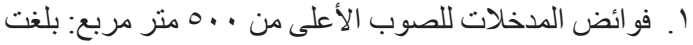

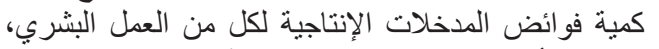

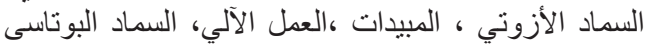

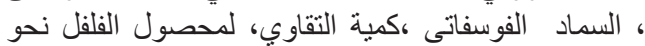
، الرهاد

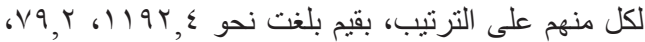
(جنيهاً

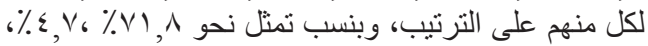

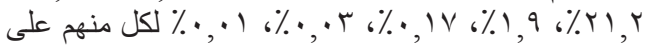

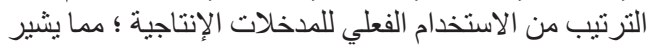

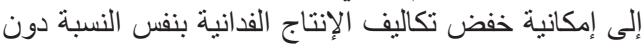

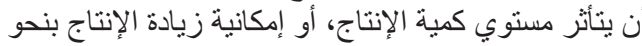

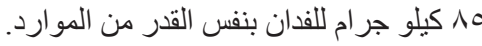

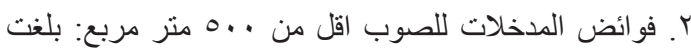

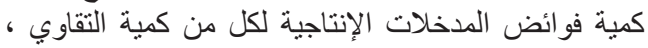

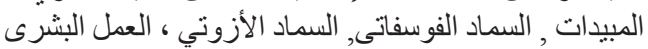

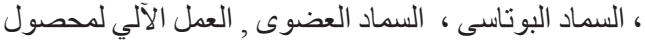

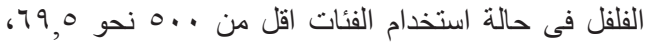

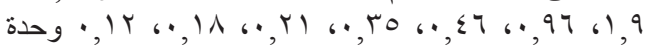

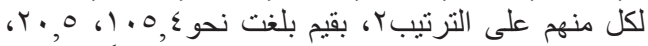

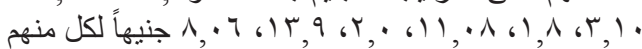

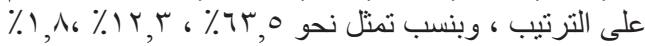

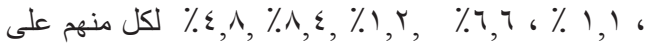

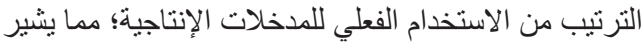

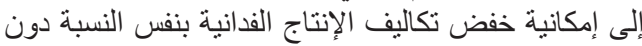

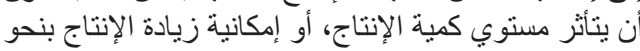

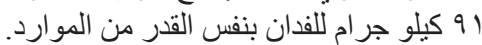

بتقدير القيم الفعلية والمستهدفة للمخرجات الإنتاجية لإنتاج

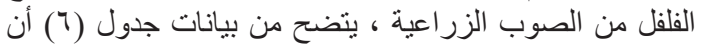

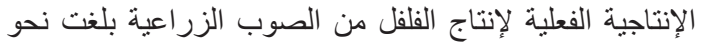

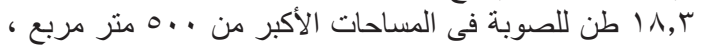

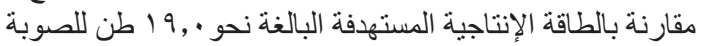

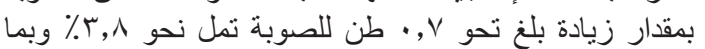

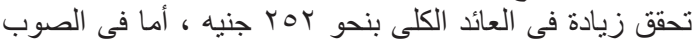
ذات المساحة الأقل من فيادة . .0 متر مربع فكانت الإنتاجية الفعلية تتساوى مع المستهدفة.

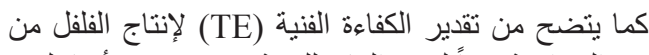

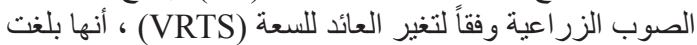

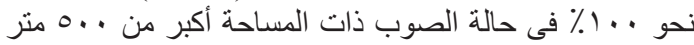

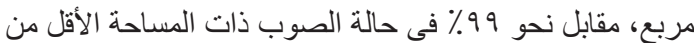

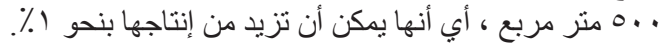

أما فيما يتعلق بتقدير كفاءة السعة (SE) لإنتاج الفلفل من

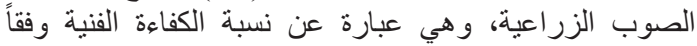

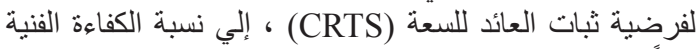

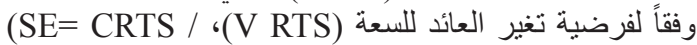
ذات ل VRTS)

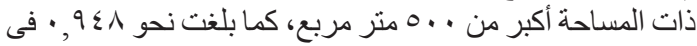

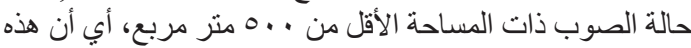

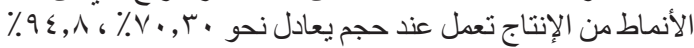

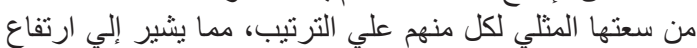

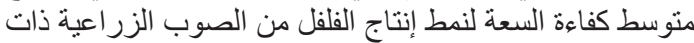

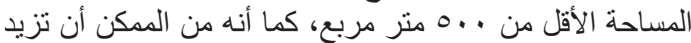

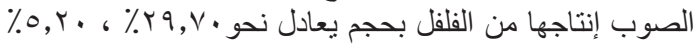
لكل منهم على الترتب حتى تصل كفاءة سعتها للو احد الصحيح. ب. تقدير الكفاءة التوزيعية لإنتاج الفلفل من الصوب الزراعية

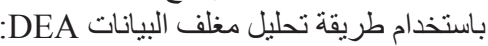

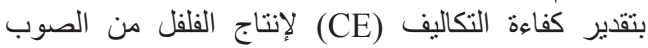

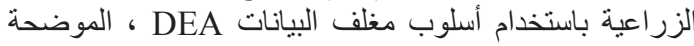

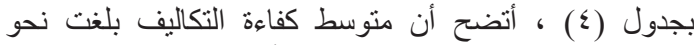

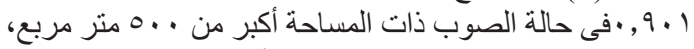

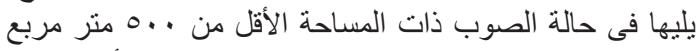

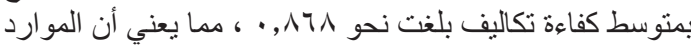

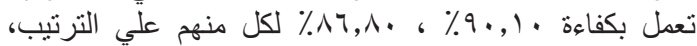

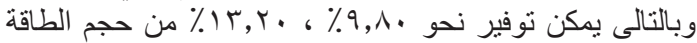

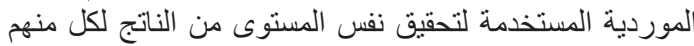

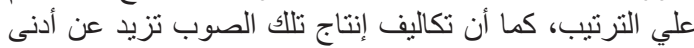

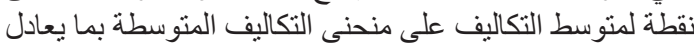
99

وفيما يتعلق بتقدير الكفاءة التوزيعية (AE) لإنتاج الفلفل

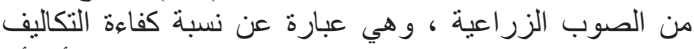

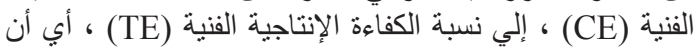

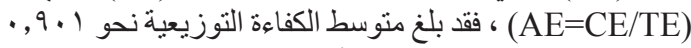

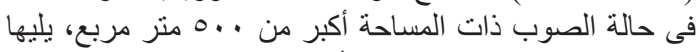

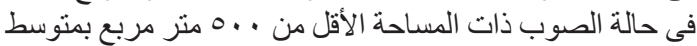

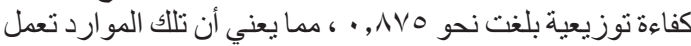

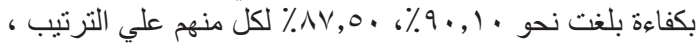

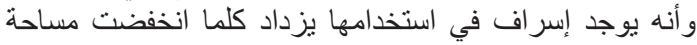

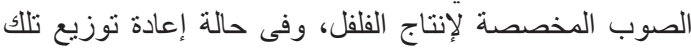

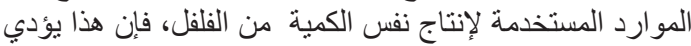

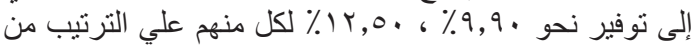

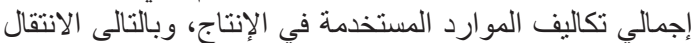

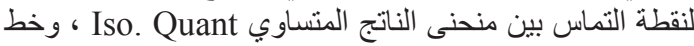

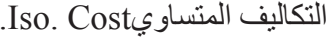

ج. تقدير الكفاءة الإقتصادية لإنتاج الفلفل من الصوب الزبات الزباتية

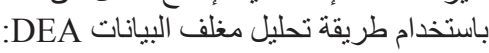

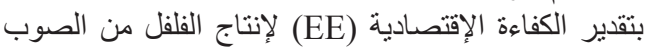

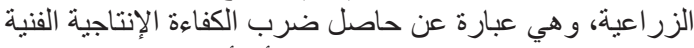

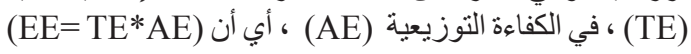




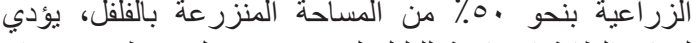

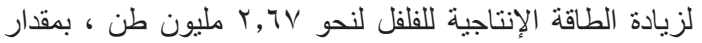

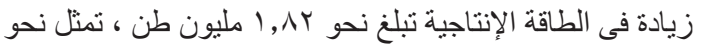

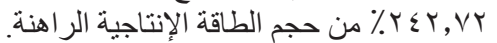

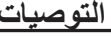

ا . ضرورة التوسع فى إنشاء الصوب الزراعية لإنتاج الفلفل

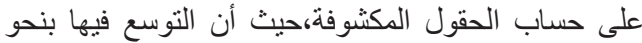

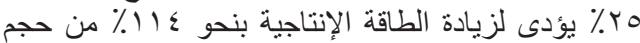

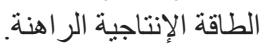

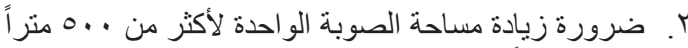

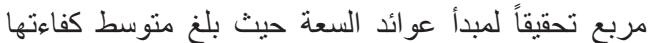

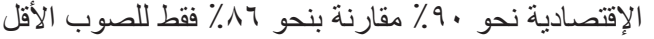

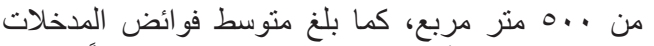

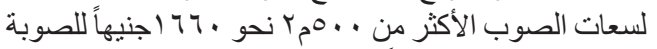

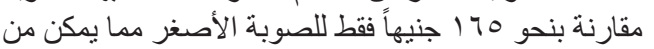
إعادة توزيع فو ائض هذة المدخلات.

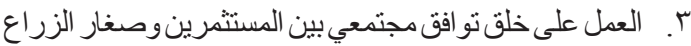
لضمان التوسع فى إقامة الصوب كضمانة للأمن الغذائي.

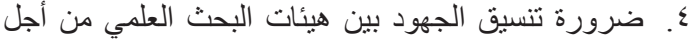

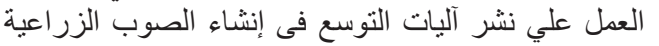

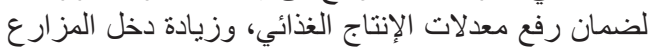
بما يحقق لله مستوى من الرفاهية.
خامسا: الوضع المستقبلي للتوسع فى إنشاء الصوب الزراعية لإنتاج الفلفل على حساب الحقول المكثوفئة

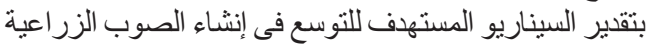

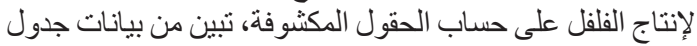

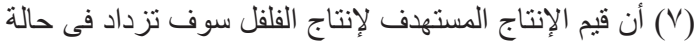

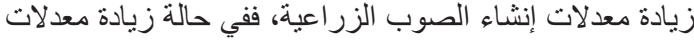

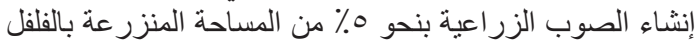

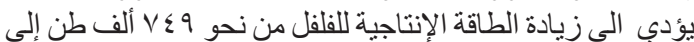

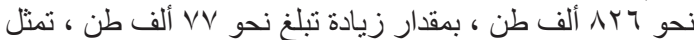
نحو ^ץ, • 1 ٪ من حجم الطاقة الإنتاجية الر اهنة.

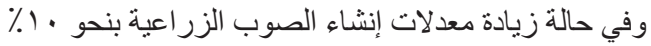
من المساحة المنزر عة بالفلفل، يؤدي الى الى زيادة الطاة الطاقة الإنتاجية

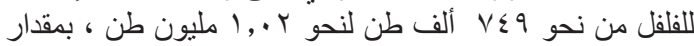

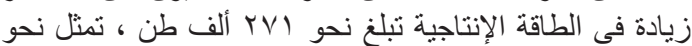

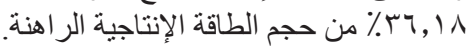

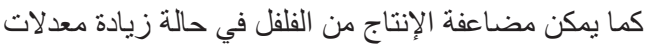

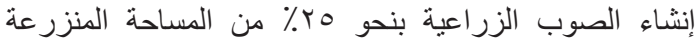

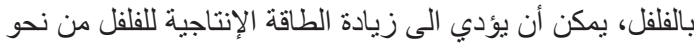

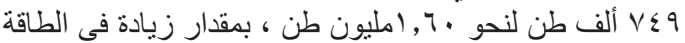

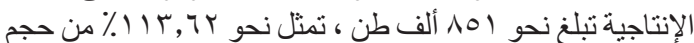

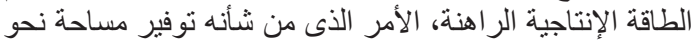

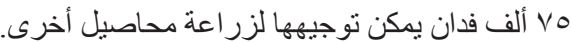

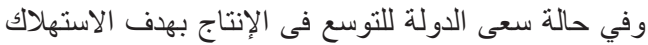

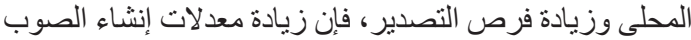

جدول ه. متوسط كمية وقيمة الفائض لمدخلات إنتاج فئات مختلفة فى عينة الاراسةلإنتاج محصول الفلقل للصوبة

\begin{tabular}{|c|c|c|c|c|c|c|}
\hline \multicolumn{3}{|c|}{ الفئات أقل من . ه متر مربع } & \multicolumn{3}{|c|}{ الفئات الأعلى من .ه متر مربع } & \multirow{2}{*}{ المدخل الإنتاجي } \\
\hline قيمة & $\%$ & 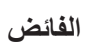 & 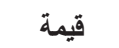 & $\%$ & الفائض ال & \\
\hline 11.08 & 6.67 & 0.35 & 1192.43 & 71.80 & 35.18 & \multirow{2}{*}{ العمل البشري(رجل/يوم) } \\
\hline 1.82 & 1.10 & 0.46 & 79.20 & 4.77 & 20.00 & \\
\hline 20.50 & 12.35 & 1.97 & 352.70 & 21.24 & 3.59 & المبيدات(جنيه) \\
\hline 8.06 & 4.86 & 0.12 & 32.91 & 1.98 & 0.46 & العمل الآلي(ساعة) \\
\hline 2.00 & 1.21 & 0.21 & 2.85 & 0.17 & 0.29 & السماد البوتاسي(وحدة) \\
\hline 3.10 & 1.87 & 0.96 & 0.43 & 0.03 & 0.25 & السماد الفوسفاتي(وحدة) \\
\hline 105.40 & 63.51 & 69.53 & 0.17 & 0.01 & 0.12 & كمية التقاوي(كجم) \\
\hline 13.99 & 8.43 & 0.18 & 0.00 & 0.00 & 0.00 & السماد العضوي(متر مكعب) \\
\hline \multicolumn{3}{|c|}{165.96} & \multicolumn{3}{|c|}{1660.69} & الإجمالي \\
\hline
\end{tabular}

المصدر : جمعت وحسبت من تحليل البيانات للعينة البحثية باستخدام برنامج

جدول 7.

\begin{tabular}{|c|c|c|c|c|c|}
\hline فيمه الزيادة فى الإيراد & 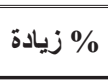 & 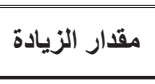 & الإنتاجية المستهدفة & الإتتاجية الفعلية & أنماط الصوب \\
\hline 252.0 & 3.8 & 0.7 & 19.0 & 18.3 & الصوب ذات المساحة أكبر من 500 متر مربع \\
\hline 0.0 & 0.0 & 0.0 & 16.1 & 16.1 & الصوب ذات المساحة الأقل من 500 مثر مربع \\
\hline
\end{tabular}

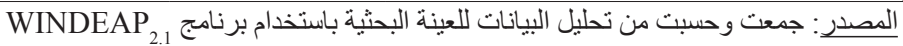

$<?>$

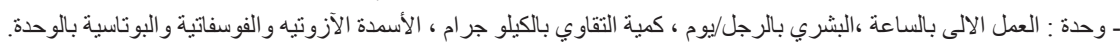

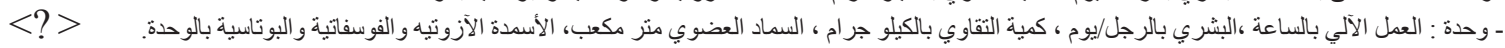

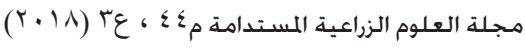


جدول رقم v. السيناريوهات المستهدفة لآثر للتوسع فى إنشاء الصوب الزراعية لإنتاج الفلفل على حساب الحقول المكشوفة

\begin{tabular}{|c|c|c|c|c|c|c|c|}
\hline \multicolumn{2}{|c|}{ مقدار الزيادة } & \multicolumn{3}{|c|}{ الإتتاج المتوقع (ألف طن) } & \multicolumn{2}{|c|}{ المساحة المتوقعة (ألف فدان) } & \multirow{2}{*}{ \% المتوقع } \\
\hline$\%$ & (ألف طن) & الإجمالى & صوب & حقل مكشوف & صوب & حقل مكشوف & \\
\hline · & . & $v \leqslant 9$ & 1 Tro & $7 \leqslant$ & r & १७ & الوضع الر اهن \\
\hline $1 \cdot, r \wedge$ & VV & Art & rro & 7.1 & 0 & 90 & 0 \\
\hline r4,11 & rVI & $1 . r^{2}$ & $\leqslant 0$. & ov. & 1. & 9. & $1 \cdot$ \\
\hline 71,90 & $\varepsilon 7 \varepsilon$ & MTK & TVO & ors & 10 & 10 & 10 \\
\hline$\Lambda V, V Y$ & $70 \mathrm{~V}$ & $1 \leqslant .7$ & $9 \ldots$ & 0.7 & $r$. & $\wedge$. & r. \\
\hline D & 101 & $17 \ldots$ & 11 ro & $\sum V_{0}$ & ro & vo & ro \\
\hline $1 \% q, r q$ & $1 \cdot \leq \varepsilon$ & IV9T & iro. & $\leq \leqslant r$ & $r \cdot$ & v. & r. \\
\hline 170,10 & IrTV & 1917 & $10 V 0$ & «1। & ro & 70 & ro \\
\hline $191, .0$ & $1 \leqslant \pi$ & riA. & $11 \ldots$ & rл. & $\varepsilon$. & 7. & $\varepsilon$. \\
\hline$r \backslash Y, \wedge r$ & $17 Y \varepsilon$ & TrVT & T. YO & $r \leqslant \Lambda$ & $\leqslant 0$ & 00 & $\leqslant 0$ \\
\hline$T \leqslant Y, V Y$ & 1111 & roTV & TrO. & TIV & 0. & 0. & 0. \\
\hline
\end{tabular}

المصدر: جمعت وحسبت من: وزارة الزر اعة واستصلاح الأر اضي: الإدارة المركزية للاقتصاد الزر اعي ـ نشرة الاقتصاد الزر اعي ـ أعداد متفرقة

Farrell,M.J., (1957) "The Measurement of Productive Efficiency", Royal Statistical Society A (General),120 (3) .

Moussa, M. Z. (1989) Econometric Estimation of Frontier Production Function and Measurement of Technical Efficiency of Corn Crop Farms in Kafr El_Sheikh Governorate", Journal of Agric Sciences, 15, No. 4, Tanta Univ.,.

Richard C. Cub, (1982) What Makes Small Business Succeed,1990.

Tim Colli,"AGuide to DEAP Version 2.1(1996): A Data Envelopment Analysis Program", Center for Efficiency and Productivity Analysis, Department of Econometrics ,University of New England.

www. alkhaleej. ae

Yen-Shogen C. (2002) Frontier Production Approaches for Measuring Efficiency of Egyptian Farms P.h.Thesis, Graduate Division, University of California.

(Received : 5 / 9 /2018; accepted:22/ 11/2018)
المراجع العربية والأجنية

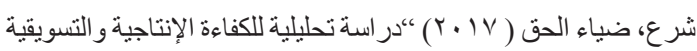

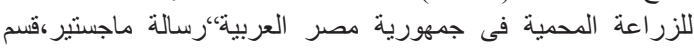
الإقتصاد الزر اعى، كلية الزر اعة، جامعة الأزهر.

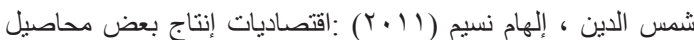

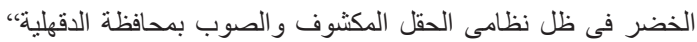

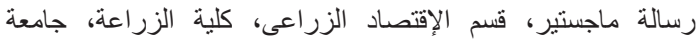
كفر الثيخ.

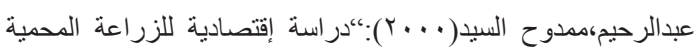

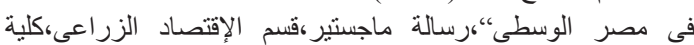

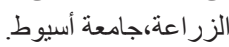

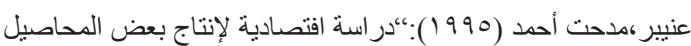

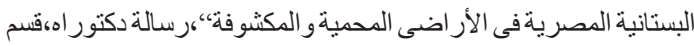

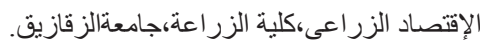

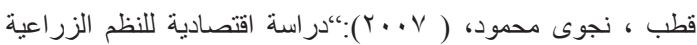

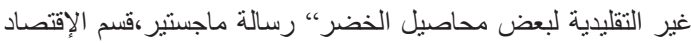
الزر اعى،كلية الزر اعة، جامعة عين شمس.

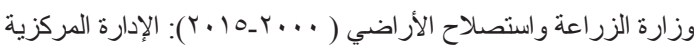
للاقتصاد الزر اعي ـنشرة الاقتصاد الزر اعي ـ أعداد متفرقة.

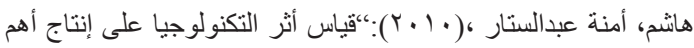

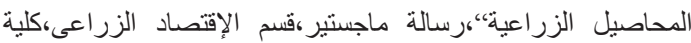

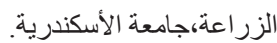




\section{Economic Study to Expand of Greenhouses On The Green Pepper Production}

Mona Tawfik, E.M. Atallah, F. Radwan and M. Z. Moussa

Department of Agricultural Economy, Faculty of Agriculture, Kafr El-Sheikh University Department of Agricultural Economy, Faculty of Agriculture, Damietta University

$\mathbf{T}$

Egypt is seeking to expand the construction of greenhouses as one of the axes of the policy of vertical expansion, For these reasons, the research paper is mainly aims at reviewing the present and expected situation of pepper greenhouse concerning average production and productivity, Qualitative and quantitative methods were utilized to achieve the study purpose, such as, probabilistic models of Data Envelopment Analysis, as well as exponential Function to estimate the growth rates, Primary data through a questionnaire of 45 greenhouses of pepper was collected from Kafr El-Sheikh governorate in addition to secondary data collected from ministry of Agriculture, The main results of the study can be Summarized as follows: The annual growth rate of greenhouse for pepper production has reached about $16 \%$ at period (2003-2015), and the average productivity of pepper greenhouse is higher than the open fields by about 6.81 once, The economic efficiency for greenhouse of pepper crop, has revealed about $91.10 \%$ for scale of more than 500 squared meters compared with $87.50 \%$ for less than 500 squared meters, Increasing the expansion of agricultural green housing to produce pepper, through increasing the acreage by about $25 \%$ will increase the production can be doubled, that will increase the production by about $114 \%$, For policy makers the study recommended the necessity to expand the numbers and acreage of the greenhouses of pepper in Egypt because they are more efficient compared with the open fields, this requires increasing the financial funds to farmers and investors.

Key Words: Greenhouse, Data Envelopment Analyses, Economic Efficiency, pepper Production 